\title{
Identification of Major Rhizobacterial Taxa Affected by a Glyphosate-Tolerant Soybean Line via Shotgun Metagenomic Approach
}

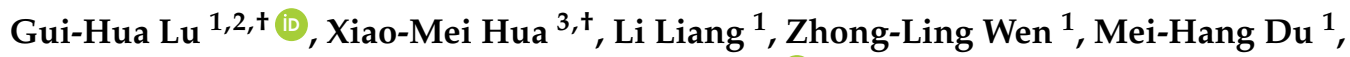 \\ Fan-Fan Meng ${ }^{4}$, Yan-Jun Pang ${ }^{1}$, Jin-Liang Qi ${ }^{1, *(\mathbb{D})}$, Cheng-Yi Tang ${ }^{1, *}$ and Yong-Hua Yang ${ }^{1, *(D)}$ \\ 1 Institute for Plant Molecular Biology, State Key Laboratory of Pharmaceutical Biotechnology, \\ School of Life Sciences, Nanjing University, Nanjing 210023, China; guihua.lu@nju.edu.cn (G.-H.L.); \\ liangliyj@163.com (L.L.); dg1730028@smail.nju.edu.cn (Z.-L.W.); 18851826162@163.com (M.-H.D.); \\ pangyj@nju.edu.cn (Y.-J.P.) \\ 2 Jiangsu Collaborative Innovation Center for Modern Crop Production, Nanjing Agricultural University, \\ Nanjing 210095, China \\ 3 State Environmental Protection Key Laboratory of Soil Environmental Management and Pollution Control, \\ Nanjing Institute of Environmental Sciences, MEP, Nanjing 210042, China; hxm@nies.org \\ 4 Jilin Academy of Agricultural Sciences, Changchun 130033, China; mengfanfan0720@163.com \\ * Correspondence: qijl@nju.edu.cn (J.-L.Q.); tangchengyi@nju.edu.cn (C.-Y.T.); yangyh@nju.edu.cn (Y.-H.Y.); \\ Tel.: +86-25-8968-6305 (Y.-H.Y.) \\ + These authors contributed equally to this work.
}

Received: 22 January 2018; Accepted: 13 April 2018; Published: 16 April 2018

\begin{abstract}
The worldwide commercial cultivation of transgenic crops, including glyphosate-tolerant (GT) soybeans, has increased widely during the past 20 years. However, it is accompanied with a growing concern about potential effects of transgenic crops on the soil microbial communities, especially on rhizosphere bacterial communities. Our previous study found that the GT soybean line NZL06-698 (N698) significantly affected rhizosphere bacteria, including some unidentified taxa, through 16S rRNA gene (16S rDNA) V4 region amplicon deep sequencing via Illumina MiSeq. In this study, we performed 16S rDNA V5-V7 region amplicon deep sequencing via Illumina MiSeq and shotgun metagenomic approaches to identify those major taxa. Results of these processes revealed that the species richness and evenness increased in the rhizosphere bacterial communities of N698, the beta diversity of the rhizosphere bacterial communities of N698 was affected, and that certain dominant bacterial phyla and genera were related to N698 compared with its control cultivar Mengdou12. Consistent with our previous findings, this study showed that N698 affects the rhizosphere bacterial communities. In specific, N698 negatively affects Rahnella, Janthinobacterium, Stenotrophomonas, Sphingomonas and Luteibacter while positively affecting Arthrobacter, Bradyrhizobium, Ramlibacter and Nitrospira.
\end{abstract}

Keywords: soil; rhizosphere; glyphosate-tolerant soybean; 16S rRNA gene; plant growth-promoting rhizobacteria; shotgun metagenomic approach

\section{Introduction}

The worldwide commercial cultivation of genetically modified (GM)/transgenic crops has increased widely during the past 20 years [1]. Cumulatively, more than 1 billion hectares of arable land have been used globally for the commercial cultivation of transgenic soybeans, especially glyphosate-tolerant (GT) soybeans [1-3]. This increased commercial cultivation of transgenic crops is accompanied by a growing concern about its potential effects on the soil microbial communities, 
especially on rhizosphere bacterial communities (reviewed in [4-6]) that play important roles in promoting plant health and growth [7-9].

Previous studies have shown differential results about the impact of transgenic plants on soil microbial communities including rhizobacterial communities. The release of many transgenic plants including certain transgenic soybean cultivars or lines has no significant effects or exerts only minor and transitory effects on soil microbial communities [10-13] (also reviewed in [4-6,14]). In other cases, the release of some transgenic plants significantly affects the composition or diversity of rhizosphere or root-endophytic microbial communities [15-18] (also reviewed in [4-6,14]).

The soil ecosystem is highly complex, and the proportion of unculturable microbes under standard lab conditions is extremely high $[19,20]$. Thus, culture-independent methods solely or combined with each other or with culture-dependent methods have been increasingly used to investigate the effect of GM/transgenic plants on soil microbiota [14]. High-throughput sequencing (hereinafter, deep sequencing) of $16 \mathrm{~S}$ rRNA gene (16S rDNA) amplicons and/or shotgun metagenome library via a next-generation sequencing (NGS) platform, e.g., 454 GS FLX pyrosequencing, is a culture-independent but powerful method that has revolutionarily facilitated soil microbiota research $[9,21-24]$. It was first applied to study microbial diversity in the deep sea [25] and also has been applied to detect the possible effects of transgenic plants on soil microbiota [11,26-28].

Meanwhile, the Illumina GAIIx [29], Illumina Hiseq, and MiSeq platforms [30,31], which are far more cost-effective than 454 GS FLX pyrosequencing, are powerful NGS technologies that have been successfully applied to study highly complicated microbial communities [32-36]. Subsequently, Illumina MiSeq has also been applied to study the effect of some transgenic plants on microbial communities [18,37-39].

Recently, shotgun metagenome sequencing (SMS) via a NGS platform combined with bioinformatics tools has been applied to deeply study the microbial community composition, structure, diversity, and/or function in plant fiber incubated in cow rumen [40], different types of soils [32], activated sludge [41], soil across nitrogen gradients [24], human gut [42], a complex biogas microbial sample from the biogas plants [43], the microbial samples of different hot springs [44,45], taproot sample of a sugar beet [46], permafrost soil [47], and other samples [48-50]. To the best of published knowledge at the web of science (http:/ / apps.webofknowledge.com, from all Databases, $118,867,781$ data limits until 12 January 2018) through searching with the combined topic words of "transgenic/genetically modified" and "metagenome/metagenomic/metagenomics", the effects of transgenic plants on soil or rhizosphere microbial communities have been rarely studied via SMS $[17,51,52]$.

Our previous study showed via $16 \mathrm{~S}$ rDNA V4 region amplicon deep sequencing by Illumina MiSeq that the GT soybean line N06-698 (hereinafter, N698) significantly affects the relative abundances of some rhizosphere bacteria. In specific, systematic contrast analysis revealed that the relative abundance of the special but unidentified operational taxonomic unit (OTU) is remarkably less than 132-fold in the rhizospheric soils of the GT line N698 compared with its control cultivar Mengdou12 (hereinafter, MD12) [18]. In the present study, we performed $16 \mathrm{~S}$ rDNA V5-V7 region amplicon via Illumina MiSeq and shotgun metagenomic approaches to identify major rhizobacterial taxa affected by the GT soybean line N698.

\section{Materials and Methods}

\subsection{Plant Materials, Field Design and Sampling}

The GT soybean line N698 and its control soybean cultivar MD12 used in this study were described in detail in our previous study [18]. In brief, the GT soybean line N698 was bred by crossing the GT soybean line NZL02-92 containing the CP4-EPSPS gene to MD12 and then the GT F1 plants continuously backcrossing to MD12 with two times. Additionally, the female and male parents of NZL02-92 were the conventional soybean cultivar Mengdou13 and the derivative strain of the GT 
transgenic soybean line AG4501 (See also Patent No. is US5998704-A) that was bred by Asgrow seed Company (Kalamazoo, MI, USA) which was merged to Monsanto company (Creve Coeur, MO, USA), respectively.

Field design and sampling were also described in detail in our previous study [18]. In brief, six sampling points per cultivar were available, and at each sampling point, two soybean plants of N698 or MD12 with adhering surrounding soil were dug out and immediately taken to the laboratory. The soil loosely adhered to soybean plant roots was shaken off and mixed as surrounding soils. Then, the soil tightly adhering to the root surface was brushed off as the rhizospheric soil. The rhizospheric soil collected from soybean plant roots from every two sampling points were mixed together as one biological replicate, and three rhizospheric soil samples were stored in a $-80^{\circ} \mathrm{C}$ freezer.

\subsection{DNA Extraction from Rhizospheric Soil Samples}

The extraction of metagenomic DNA from rhizospheric soil was described in detail in our previous study [18]. In brief, the metagenomic DNA was extracted from approximately $2 \times 0.60 \mathrm{~g}$ soil of every biological replicate by using the PowerSoil DNA Isolation Kit (MoBio Laboratories Inc., Carlsbad, CA, USA) in duplicate as recommended by the manufacturer's instructions but with minor modifications.

\subsection{Analyses of $16 S$ rDNA via Deep Sequencing Amplicons}

\subsubsection{PCR Amplification of $16 \mathrm{~S}$ rDNA (V5-V7) and Illumina MiSeq Sequencing}

Our strategy is an improved dual-index sequencing approach with paired-end (PE) $250 \mathrm{nt}$ via Illumina MiSeq [53]. The protocol was described in detail in our previous study [18] but with PE $300 \mathrm{nt}$ and the gene-specific primer for amplifying the V5-V7 region of $16 \mathrm{~S}$ rDNA, which were 799F (5'-AACMGGATTAGATACCCKG-3') and 1193R (5'-ACGTCATCCCCACCTTCC- $\left.{ }^{\prime}{ }^{\prime}\right)$. The specific primer pair was selected due to the previous study by Bulgarelli et al. [22] and Schlaeppi et al. [54]. Additionally, the qualified metagenomic DNA of each sample was normalized to $30 \mathrm{ng}$ per PCR reaction within a $50 \mu \mathrm{L}$ volume. 16S rDNA V5-V7 amplicon deep sequencing clean data of 6 samples have been submitted to the NIH Sequence Read Archive (SRA), and the SRA accession is SRP136046.

\subsubsection{Operational Taxonomic Unit Selection and Analysis of Species Composition and Abundance}

OTU selection and analysis of species composition and abundance were described in detail in our previous study [18] with minor corrections by using the software UPARSE [55] implemented as the cluster_OTU command in USEARCH (v7.0.1090) [55]. Furthermore, the OTU counts in each sample's library were normalized using rarefaction corresponding to the sample with the least absolute filtered tags at $97 \%$ similarity in the group after species annotation was performed and a phylogenetic tree was constructed.

\subsubsection{Alpha and Beta Diversity Analyses}

Alpha and beta diversity analyses were also described in detail in our previous study [18]. Briefly, principal coordinate analysis (PCoA) was drawn by software R package v3.1.3 (R Development Core Team) to exhibit the differences between the groups according to the matrices of beta diversity distances calculated by QIIME (v1.8.0) [56].

\subsection{Shotgun Metagenomic Approaches}

\subsubsection{Construction of Metagenomic DNA Library}

Shotgun metagenomic DNA library was constructed in accordance with the manufacturer's instructions (Illumina, CA, USA) [57] with some minor modifications. In brief, three metagenomic DNA samples each with $0.4 \mu \mathrm{g}$ DNA taken from MD12 or N698 rhizospheric DNA sample were pooled as one qualified sample for shotgun metagenome sequencing, named MGMRh or MGNRh. 
Exactly $1.2 \mu \mathrm{g}$ qualified DNA of MGMRh or MGNRh sample in $80 \mu \mathrm{L}$ TE buffer was sheared into small fragments less than 600 bp by nebulization. Afterwards, the $3^{\prime}$ end of the phosphorylated blunt DNA fragments was added with an adenine (A) base, and then ligated with Illumina adapter oligo mix. Furthermore, the adapter-modified DNA fragments were magnified by NEB Phusion high-fidelity PCR master mix (New England Biolabs, MA, USA) with $65^{\circ} \mathrm{C}$ melting temperature and 12 cycles. Moreover, adapted DNA fragments of 400-600 bp were purified by QIAquick PCR purification kit (Qiagen, Shanghai, China), and then qualified by Agilent 2100 Bioanalyzer (Agilent Technologies, CA, USA) and quantified by ABI StepOnePlus Real-Time PCR System (Applied Biosystem, CA, USA). The PE shotgun metagenomic libraries were constructed with inserted fragment for the MGMRh and MGNRh samples.

\subsubsection{Shotgun Metagenome Sequencing}

The qualified metagenomic libraries were deeply sequenced with the PE $125 \mathrm{nt}$ strategy via the Illumina HiSeq2500 NGS platform (Illumina, CA, USA) and HiSeq PE Cluster Kit v4 (Illumina, CA, USA) by BGI Tech Solutions Co., Ltd. (Shenzhen, China). Shotgun metagenome sequencing clean data of MGMRh and MGNRh samples have also been submitted to SRA, and the SRA accession is SRP136046 too.

\subsubsection{Quality Control and De Novo Metagenome Assembly}

Clean data were obtained after sequencing adapters, and reads with ambiguous $\mathrm{N}$ base or average base quality score less than 15 were removed from raw data. De novo metagenome assembly was performed with IDBA-UD (v1.1.1) [58] for each sample, and reads were assembled with a series of different $k$-mer size (25-115 bp) in parallel, and then were mapped back to each of the assembled contigs for validation. The best assembly was selected based on contig N50 and mapping rated, and only those contigs that were longer than $500 \mathrm{bp}$ were kept for further analysis.

Metagenomic analysis was also performed by One Codex data platform (hereinafter, One Codex) [59] with cloud-based $k$-mer method, after the compressed FASTQ data, which was appended with .gz, was uploaded to the One Codex platform [59], considering that One Codex is more accurate than either the MG-RAST or the Kraken tools [60].

\subsubsection{Taxonomic Assignment}

Based on the known sequence database of bacteria, fungi and archaeobacteria retrieved from the nucleotide database of the National Center of Biotechnology Information (NCBI, GenBank Flat File Release 201.0, until 31 May 2014) including 1,099,685 bacterial sequence entries, clean reads of each sample were aligned by SOAPaligner (v2.21, also named as SOAP2) [61]. Then, mapped clean reads were assigned to the corresponding taxon and summarized. Taxonomic assignment was also performed by One Codex based on the One Codex database (hereinafter, One Codex DB) and the NCBI RefSeq Complete Genomes database (hereinafter, RefSeq DB). One Codex DB contains 37,183 microbial genomes until December 2017, including 30,825 bacterial, 5163 viral, 633 fungal, 504 archaeal, and 57 protozoan genomes, which collect 29,063 more genomes than RefSeq DB (https: / / app.onecodex.com/references). RefSeq DB has 8120 microbial genomes, including 2948 bacterial, 4726 viral, 264 fungal, and 181 archaeal reference and representative genomes downloaded from the NCBI until December 2017 at the One Codex data platform (https:/ / app.onecodex.com/references).

\subsection{Statistical Analyses}

Wilcoxon rank-sum test was used to examine the significance of alpha diversity indices. Metastats [62], described in detail in our previous studies [18,63], was used to obtain the relative abundance differences of microbial communities between groups (groups $=2$, samples per group $\geq 3$ ). The obtained $p$-value was adjusted by Benjamini-Hochberg false discovery rate (FDR) [64] correction (function "p.adjust" in the stats package of $\mathrm{R}(\mathrm{v} 3.1 .3)$ ). 


\section{Results}

3.1. Composition and Structure of Rhizosphere Bacterial Community Revealed by 16S rDNA V5-V7 Amplicon Deep Sequencing

3.1.1. Overall Analysis of $16 \mathrm{~S}$ rDNA (V5-V7 Hypervariable Region) Amplicon Sequencing Data-Based Illumina MiSeq

A total of 413,978 qualified pairs of clean reads (300 nt average) were obtained with an average of 68,996 (range: 40,515-106,532) per rhizospheric soil sample of the GT soybean line N698 (NRh) and that of its control cultivar MD12 (MRh). Then, 130,813 filtered tags (417 $\pm 4 \mathrm{nt}$ ) at 97\% similarity were obtained with an average of 21,802 (range: 13,416-32,720) per sample. Next, the OTU counts were normalized using rarefaction corresponding to the filtered tags of NRh1 (Table S1). Finally, 5144 OTUs except singletons were identified with an average of 857 OTUs per rhizospheric soil sample (Table S1) and were summarized with taxonomic annotation in Table S2. All OTU sequences were shown in File S1.

\subsubsection{Alpha Diversity of Bacterial Communities in Rhizospheric Soil}

The rarefaction curves of the observed OTU number, Chao 1, abundance coverage-based estimator (ACE) and Shannon of rhizospheric soil samples were calculated based on the normalization of OTU counts. All curves nearly reached the saturation plateau (Figure S1), indicating that the sequencing depth included sufficient detectable species in bacterial communities and was sufficient to capture the diversity of bacterial communities in those samples. The mean and standard deviation of five alpha diversity indices of the rhizospheric soil groups were then calculated (Table S3). All p-values of five indices of alpha diversity in Table S3 were higher than 0.05 between the NRh and MRh samples as calculated by Wilcoxon rank-sum test, (Table S3). This result indicates no significant difference in the overall indices of the alpha diversity. However, all five indices of alpha diversity of the rhizospheric soil of N698 were separated from that of MD12 (Figure 1) when boxplot analysis was used to visualize the differences.
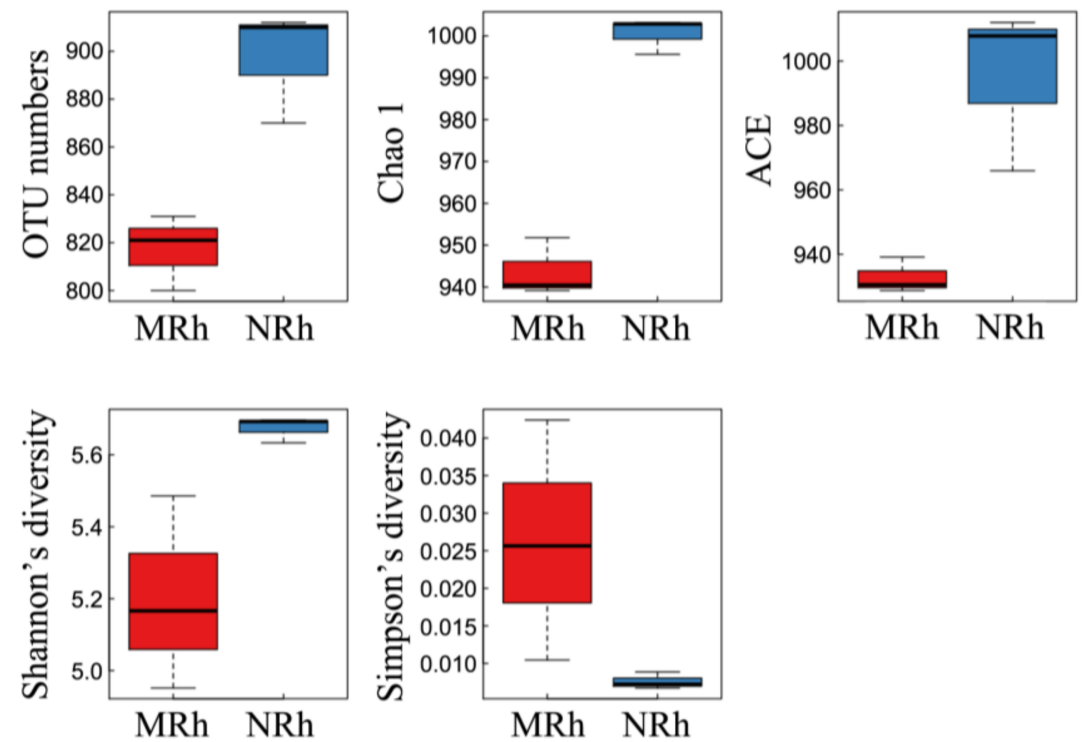

Figure 1. Boxplot analysis of five indices of alpha diversity. Five lines from top to bottom are the maximum value, the third quartile, median, the first quartile, and the minimum value. MRh represents the rhizospheric soil samples of the control soybean cultivar MD12 (n = 3). NRh represents the rhizospheric soil samples of the glyphosate-tolerant (GT) soybean line N698 (n=3). OTU: operational taxonomical unit; ACE: abundance coverage-based estimator. 


\subsubsection{Beta Diversity of Bacterial Community in the Rhizosphere Soil}

Principal component analysis (PCA) displayed that the bacterial communities in the rhizospheric soil of the transgenic line N698 were distinct from those of MD12 (Figure 2A). Then, phylogenetic beta diversity analysis was performed by PCoA based on the unweighted uniFrac (UUF) and weighted uniFrac (WUF) distance metrics. The bacterial communities in the rhizospheric soil of N698 were distinct from those in the rhizospheric soil of MD12 (Figure 2B,D). Furthermore, taxonomic beta diversity analysis was performed by PCoA based on the Bray-Curtis distance metrics. Here the bacterial communities in the rhizospheric soil of N698 were distinct from those in the rhizospheric soil of MD12 (Figure 2C).
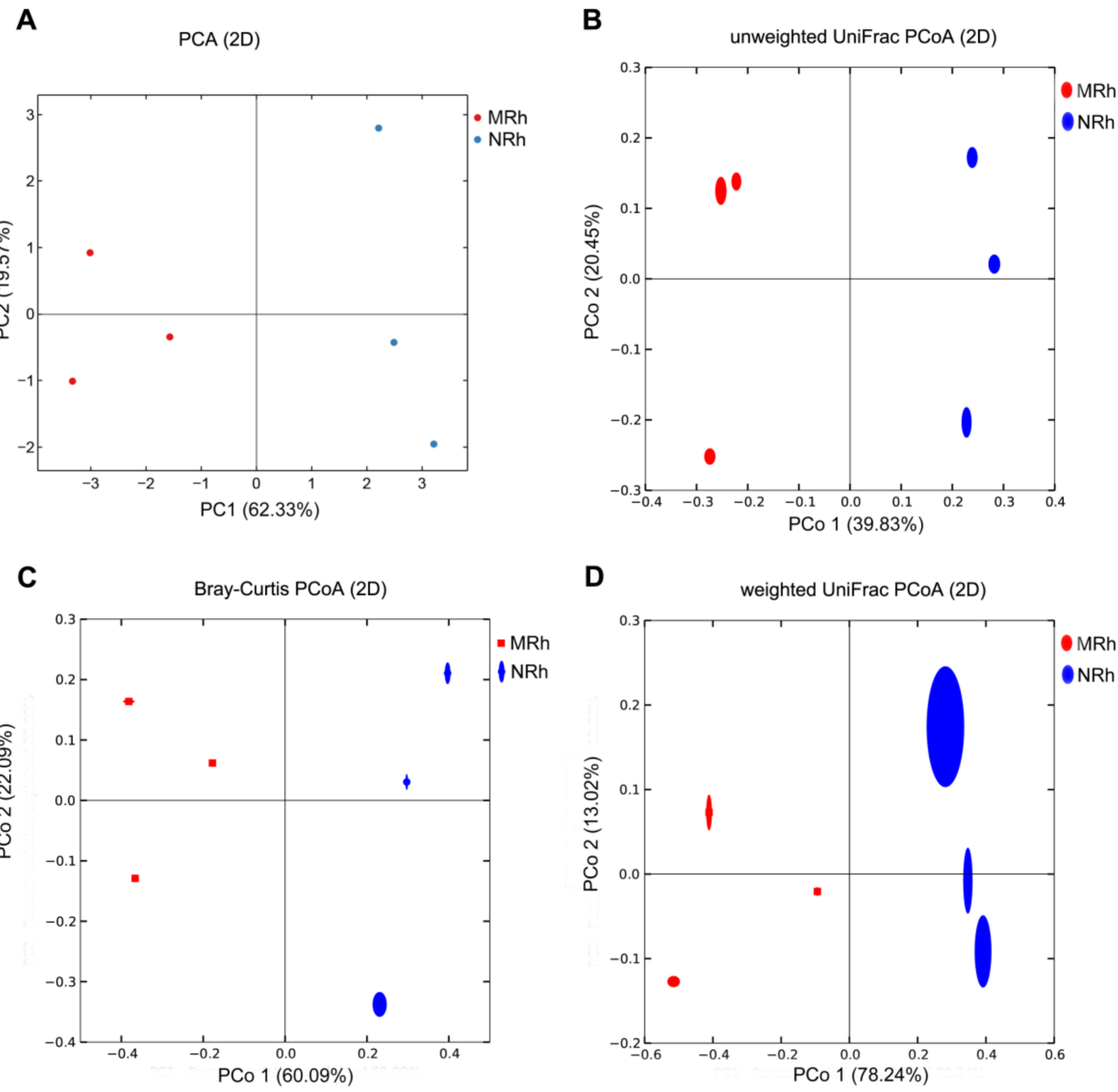

Figure 2. Beta diversity analysis of the rhizosphere bacterial communities between N698 and MD12 $(n=6)$. Principal component analysis (PCA) based on OTU abundance of bacterial communities (A); Numbers in brackets represent contributions of principal components to the total variance; The red and light blue dots represent rhizospheric soil replicates of MD12 (MRh) and those of N698 (NRh), respectively. Principal coordinate analysis (PCoA) based on unweighted UniFrac metrics (B), Bray-Curtis metrics (C), and weighted UniFrac metrics (D); the variance explained by each principal coordinate axis is shown in PCo 1 vs. PCo 2; Red ovals or squares represent MD12, and blue ovals represent N698. 


\subsubsection{Comparison of Dominant Bacterial Phyla in the Rhizospheric Soil between N698 and MD12}

The taxonomic composition and distribution of the rhizospheric soil of N698 and MD12 at the phylum level (Table S4) showed that the most abundant phylum was Proteobacteria, followed by Actinobacteria, Acidobacteria or Gemmatimonadetes, and then by Bacteroidetes or Verrucomicrobia and Firmicutes (Table S4). In addition, the relative abundance of Proteobacteria was significantly $(p=0.0163)$ lower in the rhizospheric soil of N698 than that of MD12 (Table S4).

\subsubsection{Comparison of Dominant Bacterial Genera in the Rhizospheric Soil between N698 and MD12}

A total of 286 genera were detected in the rhizospheric soil of N698 and MD12 (Table S5), and the relative abundances of 36 among 100 characterized genera were significantly $(p<0.05)$ different between the rhizosphere of N698 and MD12 except those unclassified genera (Table S6). Surprisingly, both Yersinia and Serratia were not found in the normalized OTU table for biom format (Table S2) by 16S rDNA V5-V7 amplicon deep sequencing, although Yersinia or Serratia was detected by 16S rDNA V4 amplicon deep sequencing. Moreover, we compared the top 30 dominant rhizobacterial genera of N698 and MD12 revealed by 16S rDNA V5-V7 and V4 region amplicon deep sequencing (Table S8) and visualized the top 10 dominant genera in Figure 3A,B. Among those dominant genera, Rahnella, Variovorax, Ewingella and Ramlibacter were detected only by 16S rDNA V5-V7 region amplicon deep sequencing. By contrast, Yersinia/Serratia, Pedobacter, Luteibacter and Flavisolibacter were detected only by $16 \mathrm{~S}$ rDNA V4 region amplicon deep sequencing (Table S8, Figure 3). We were unable to identify whether the highest abundance OTU is Rahnella, Yersinia or Serratia.

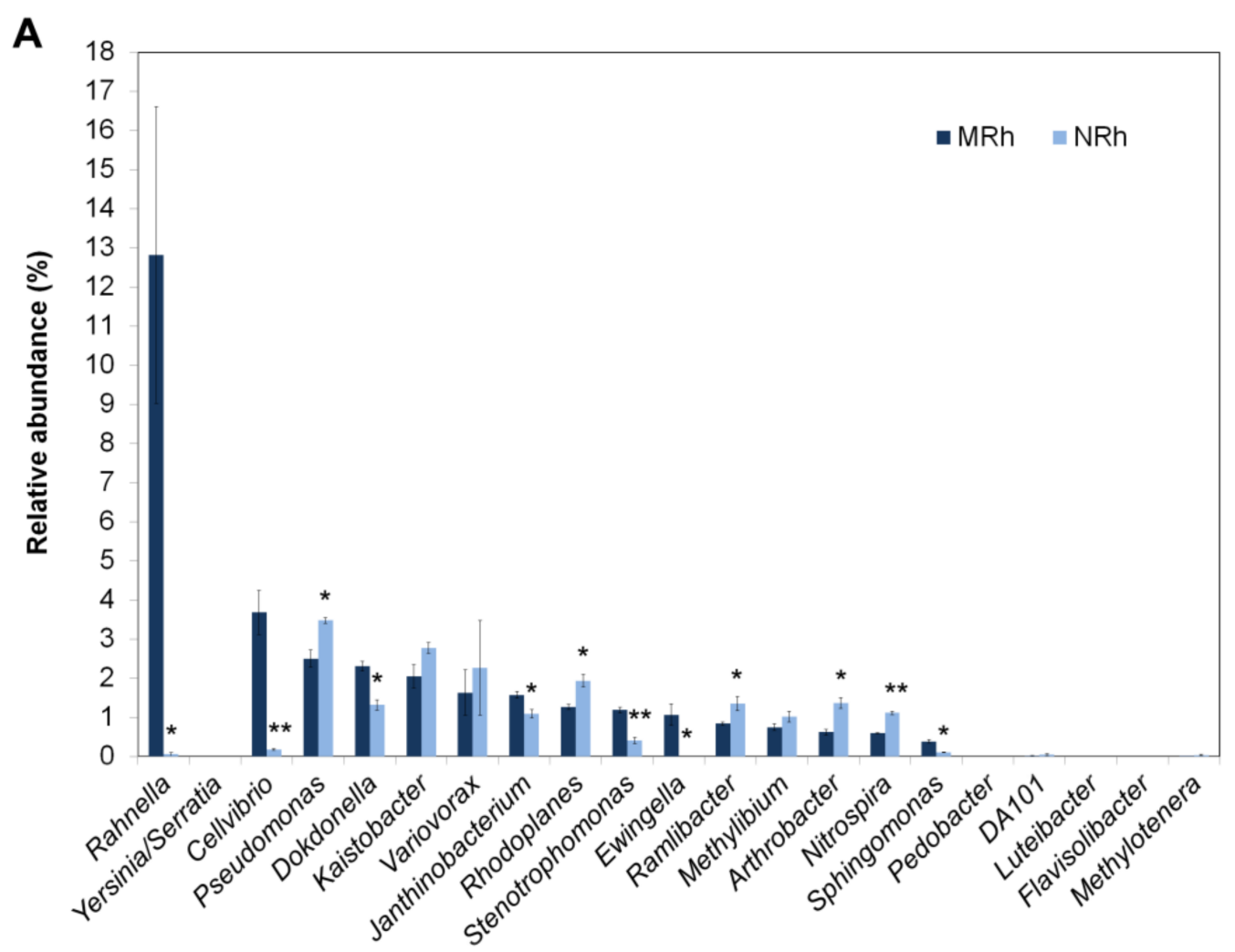

Figure 3. Cont. 

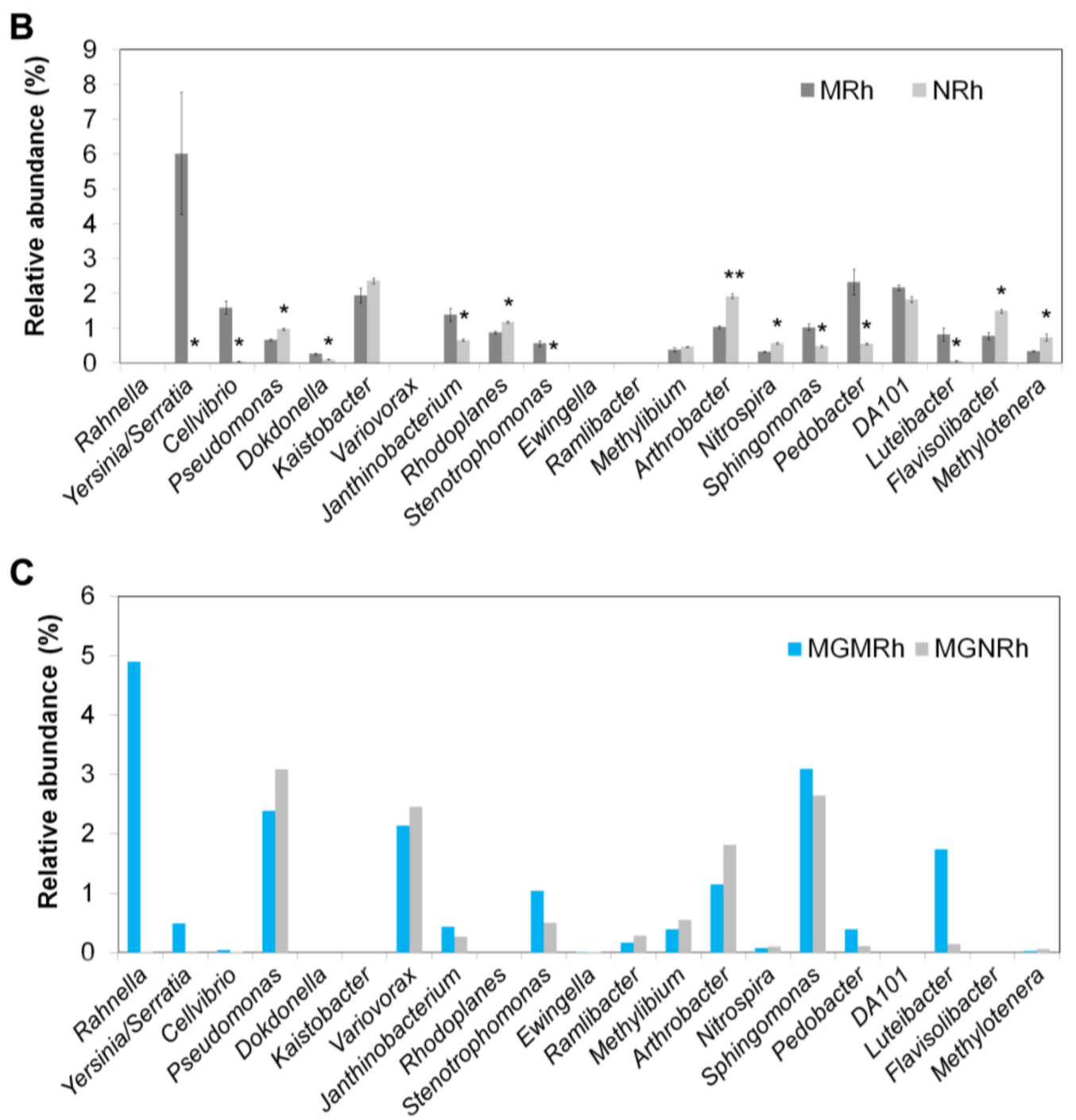

Figure 3. Comparison of the relative abundances of top 10 dominant genera in the rhizospheric soil between N698 and MD12. (A) Results revealed by 16S rDNA V5-V7 hypervariable region amplicon deep sequencing. (B) Results revealed by $16 \mathrm{~S}$ rDNA V4 hypervariable region amplicon deep sequencing. (C) Results revealed by shotgun metagenomic approaches (analyzed by One Codex). Error bars indicate standard errors; * $p<0.05 ;{ }^{* *} p<0.01$. MRh1-3 and NRh1-3 represent three biological rhizospheric soil replicates of MD12 (MRh) and those of N698 (NRh), respectively. MGMRh and MGNRh represent pooled rhizospheric DNA sample of MRh and NRh for shotgun metagenome sequencing, respectively.

Furthermore, we blasted the 418 bp $16 \mathrm{~S}$ rDNA V5-V7 region sequence of OTU (File S1) with the highest abundance and found that the OTU should be Rahnella aquatilis (Table S7). However, the OTU was $99 \%$ similar (416/417 bp) not only to the $16 \mathrm{~S}$ rDNA of Rahnella sp. G7/Y2/Z2-S1, R. aquatilis strain JS119, but also to the $16 \mathrm{~S}$ rDNA of Serratia sp. THG-CN21 via basic local alignment search tool (BLAST). Furthermore, the OTU was $99 \%$ similar $(415 / 417 \mathrm{bp})$ to the genome fragment of R. aquatilis CIP 78.65 (ATCC 33071) via BLAST. In our previous study, the $253 \mathrm{bp} 16 \mathrm{~S}$ rDNA V4 region sequence of OTU with the highest abundance in the rhizobacterial community of N698 was 100\% similar not only to the $16 \mathrm{~S}$ rDNA of Yersinia pestis CO92, Yersinia enterocolitica subsp. enterocolitica 8081, and Serratia proteamaculans 568 , but also to the $16 \mathrm{~S}$ rDNA of Rahnella sp. strain S04, R. aquatilis strain YHBT21/YHBT11 and to the genome fragment of Serratia plymuthica S13 after BLAST [18]. To date, the highest abundance OTU in the present study was still not identified.

Thus, we performed SMS and bioinformatics analysis to deal with this issue. 


\subsection{Composition of Rhizosphere Microbial Communities Revealed by Shotgun Metagenomic Approaches}

\subsubsection{Statistical Summary of Assembled Shotgun Metagenome Sequencing Data}

On average, 58,728,828 clean reads and 7.34 Gbp clean data per sample were generated from SMS (Table S9). The total clean reads of the MGMRh and MGNRh samples were assembled by IDBA-UD (v1.1.1) first, but their mapping rates were only $0.983 \%$ and $2.368 \%$, respectively (Table S9). Thus, de novo metagenome assembly of two samples was reperformed with One Codex, which displayed an obvious increase to more than $8.23 \%$ of MGMRh or $6.97 \%$ of MGNRh in the mapping rates of both samples based on the RefSeq complete genome database (Table S9).

3.2.2. Computation and Comparison of Taxonomic Assignment between MGMRh and MGNRh by SOAPaligner Based on the NCBI Nucleotide Database

Taxonomic assignment was performed via SOAPaligner by aligning clean reads of the MGMRh or MGNRh sample directly to the NCBI nucleotide database, and then mapped clean reads were assigned to the corresponding taxons and summarized (Table S10, sheets 1-6). On one side, the genus with the most mapped read count (316,533 reads) in the MGMRh sample was Rahnella (Table 1, sheet 5 of Table S10), and its relative abundance was more than $40 \%$ of the total mapped reads and was consistent with the result revealed by $16 \mathrm{~S}$ rDNA V5-V7 amplicon deep sequencing (Table S6). By contrast, the mapped read count of Rahnella in the MGNRh sample was only 385 reads and $0.121 \%$ of the total mapped reads (Table 1, sheet 5 of Table S10). This finding is consistent with the result revealed by $16 \mathrm{~S}$ rDNA V5-V7 amplicon deep sequencing (Table S6). Serratia was detected as the top four genus in the MGMRh sample, although it was detected with much less mapped reads in the MGNRh sample. Yersinia was also detected but with few mapped reads either in the MGMRh or MGNRh sample (Table 1, sheet 5 of Table S10). Additionally, Variovorax, Ramlibacter, Pedobacter, Luteibacter and Flavisolibacter were detected by SOAPaligner in either the MGMRh or MGNRh sample (Table 1, sheet 5 of Table S10).

Table 1. Identification and comparison of special genera in the rhizospheric soil of the glyphosate-tolerant (GT) soy bean line N698 and its control MD12 by shotgun metagenomic approaches.

\begin{tabular}{|c|c|c|c|c|c|c|}
\hline \multirow{4}{*}{$\begin{array}{c}\text { Bioinformatics Tool } \\
\text { Database } \\
\text { Sample name } \\
\text { Total mapped reads }\end{array}$} & \multirow{2}{*}{\multicolumn{2}{|c|}{$\begin{array}{c}\text { SOAPaligner } \\
\text { NCBI nucleotide database }\end{array}$}} & \multicolumn{4}{|c|}{$\begin{array}{c}\text { One Codex Data Platform } \\
\end{array}$} \\
\hline & & & \multicolumn{2}{|c|}{ One Codex DB } & \multicolumn{2}{|c|}{ RefSeq DB } \\
\hline & MGMRh & MGNRh & MGMRh & MGNRh & MGMRh & MGNRh \\
\hline & 790,310 & 318,187 & $7,413,129$ & $6,118,123$ & $4,878,689$ & $4,058,863$ \\
\hline Genus name & \multicolumn{6}{|c|}{ Mapped reads (proportions of total mapped reads) } \\
\hline Rahnella ${ }^{1}$ & $316,533(40.052 \%)$ & $385(0.1210 \%)$ & $363,110(4.898 \%)$ & $510(0.00834 \%)$ & $216,768(4.443 \%)$ & $364(0.00897 \%)$ \\
\hline Serratia & $28,900(3.657 \%)$ & $38(0.0120 \%)$ & $36,565(0.4932 \%)$ & $1416(0.02314 \%)$ & $20,940(0.4293 \%)$ & $1959(0.04826 \%)$ \\
\hline Ewingella $^{1}$ & ND & ND & $1077(0.01453 \%)$ & $67(0.00110 \%)$ & ND & ND \\
\hline Ramlibacter $^{1}$ & $1846(02336 \%)$ & $2518(0.7913 \%)$ & $12,642(0.1705 \%)$ & $17,414(0.28463 \%)$ & $17,680(0.3624 \%)$ & $23,159(0.57058 \%)$ \\
\hline Luteibacter $^{2}$ & $24(0.0030 \%)$ & $2(0.0006 \%)$ & $129,081(1.7413 \%)$ & $9029(0.14758 \%)$ & ND & ND \\
\hline Pedobacter ${ }^{2}$ & $638(0.0807 \%)$ & $166(0.0522 \%)$ & $29,399(0.3966 \%)$ & $6812(0.11134 \%)$ & $5210(0.1068 \%)$ & $2026(0.04991 \%)$ \\
\hline Flavisolibacter $^{2}$ & $20(0.0025 \%)$ & $19(0.0060 \%)$ & ND & ND & ND & ND \\
\hline
\end{tabular}

Limited total mapped clean reads were identified via SOAPaligner, i.e., only 790,310 of $59,269,260$ clean reads in the MGMGh sample and 318,187 of 58,188,396 clean reads in the MGNRh sample (Table 1, Table S10). Thus, we performed computation and comparison of taxonomic assignment by using another bioinformatics tool, One Codex. 
3.2.3. Computation and Comparison of Taxonomic Assignment between MGMRh and MGNRh by One Codex Based on One Codex DB

We uploaded our PE SMS files of the MGMRh and MGNRh samples by using Windows Uploader provided by Minot et al. [59] because we cannot upload our SMS files via One Codex command-line-interface. Basing on One Codex DB, we classified $12.43 \%$ of $29,634,630$ clean reads in Reads_MGMRh_1.fq.gz (MGMRh_1), 12.29\% of 29,634,630 clean reads in Reads_MGMRh_1.fq.gz (MGMRh_2), 10.47\% of 29,094,198 clean reads in Reads_MGNRh_1.fq.gz (MGNRh_1), and 10.34\% of 29,094,198 clean reads in Reads_MGNRh_1.fq.gz (MGNRh_2) as mixed metagenomic samples at all taxonomic levels by using One Codex (Table S11). On the one hand, the genus with the most mapped read count in the MGMRh sample was Rahnella, with a relative abundance of $4.898 \%$ of the total mapped reads (Table S12 and Table 1, and Figure 3C). On the other hand, Rahnella in the MGNRh sample had only 510 mapped reads, which was only $0.00834 \%$ of the total mapped clean reads (Table S12, Table 1). Both results were consistent with those analyzed by SOAPaligner and revealed by $16 \mathrm{~S}$ rDNA V5-V7 amplicon deep sequencing (Figure 3A,C). Serratia was also discovered in the MGMRh sample, but it was detected with much less mapped reads in the MGNRh sample. Meanwhile, Yersinia was found with few mapped reads either in the MGMRh or MGNRh sample (Table S12 and Table 1). Furthermore, Variovorax, Ewingella, Ramlibacter, Pedobacter and Luteibacter were detected by One Codex based on One Codex DB at the genus level in either the MGMRh or MGNRh sample (Table 1 and Table S12).

3.2.4. Computation and Comparison of Taxonomic Assignment between MGMRh and MGNRh by One Codex Based on RefSeq DB

Besides One Codex database, RefSeq DB downloaded from NCBI is available at the One Codex data platform. We further performed taxonomic assignment of MGMRh_1, MGMRh_2, MGNRh_1 and MGNRh_2 files by One Codex based on RefSeq DB. We classified $8.17 \%$ of $29,634,630$ clean reads in MGMRh_1, 8.06\% of 29,634,630 clean reads in MGMRh_2, 6.95\% of 29,094,198 clean reads in MGNRh_1, and $6.85 \%$ of $29,094,198$ clean reads in MGNRh_2 as mixed metagenomic samples at all taxonomic levels by using One Codex (Table S13). The genus with the most mapped read count in the MGMRh sample was Rahnella, with a relative abundance of $4.443 \%$ of the total mapped reads (Table S14 and Table 1). Rahnella in the MGNRh sample had only 364 mapped reads, which was only $0.00897 \%$ of the total mapped clean reads. Both results were consistent with the result analyzed by SOAPaligner and by One Codex based on One Codex DB. In addition, Serratia was also discovered in the MGMRh sample, but it was detected with much less mapped reads in the MGNRh sample. Meanwhile, Yersinia was found with some mapped reads in the MGMRh sample but with much less mapped reads in the MGNRh sample (Table S14 and Table 1). Moreover, Variovorax, Ramlibacter, Pedobacter and Luteibacter were detected in either the MGMRh or MGNRh sample, whereas Ewingella and Flavisolibacter were not detected at the genus level by One Codex based on RefSeq DB (Table 1 and Table S14).

\subsubsection{Identification of Rhizobacterial Species of Rahnella and Serratia by Shotgun Metagenomic Approaches}

At the species level, Rahnella sp. Y9602 had the most mapped read count in the MGMRh sample, and $R$. aquatilis was one of the top 10 species in the MGMRh sample revealed by SOAPaligner, but Rahnella sp. WP5 was undetected. Additionally, Serratia liquefaciens was the 3rd top species in the MGMRh sample revealed by SOAPaligner (Table 2, and Table S10 in detail). However, the mapped clean reads of all three species were few in MGNRh sample (Table 2, and Table S10 in detail).

Based on the One Codex DB, Rahnella sp. WP5 was identified as the top species with the most mapped read count in the MGMRh sample revealed by One Codex (Table 2, and Table S15 in detail). R. aquatilis also belonged to the top 10 species, and Rahnella sp. Y9602 was detected with 12,208 mapped clean reads. Meanwhile, S. liquefaciens was one of the top 10 species (Table 2, and Table S15 in detail). 
With regard to the results analyzed by SOAPaligner, the mapped clean reads of all three species were found with few counts in the MGNRh sample (Table 2, and Table S15 in detail).

Table 2. Identification and comparison of rhizobacterial species of Rahnella and Serratia in MGMRh and MGNRh by shotgun metagenomic approaches.

\begin{tabular}{|c|c|c|c|c|c|c|}
\hline \multirow{3}{*}{$\begin{array}{c}\text { Bioinformatics Tool } \\
\text { Database } \\
\text { Sample name }\end{array}$} & \multirow{2}{*}{\multicolumn{2}{|c|}{$\begin{array}{c}\text { SOAPaligner } \\
\text { NCBI nucleotide database }\end{array}$}} & \multicolumn{4}{|c|}{ One Codex Data Platform } \\
\hline & & & \multicolumn{2}{|c|}{ One Codex DB } & \multicolumn{2}{|c|}{ RefSeq DB } \\
\hline & MGMRh & MGNRh & MGMRh & MGNRh & MGMRh & MGNRh \\
\hline Total mapped reads & 790,310 & 318,187 & $7,413,129$ & $6,118,123$ & $4,878,689$ & $4,058,863$ \\
\hline \multicolumn{7}{|l|}{ Species name ${ }^{1,2}$} \\
\hline Rahnella aquatilis & 8260 & 9 & 26,556 & 46 & 216,768 & 364 \\
\hline Rahnella sp. WP5 & ND & ND & 89,230 & 86 & ND & ND \\
\hline Rahnella sp. Y9602 & 37,445 & 35 & 12,208 & 25 & ND & ND \\
\hline SUM & 45,705 & 44 & 127,994 & 157 & 216,768 & 364 \\
\hline Serratia liquefaciens & 24,395 & 25 & 32,253 & 91 & ND & ND \\
\hline Serratia proteamaculans & 267 & 0 & ND & ND & 7693 & 184 \\
\hline Serratia plymuthica & 258 & 1 & 304 & 37 & 6042 & 289 \\
\hline Serratia marcescens & 41 & 4 & 452 & 149 & 4184 & 1223 \\
\hline Serratia rubidaea & ND & ND & 142 & 134 & ND & ND \\
\hline Serratia sp. S4 & ND & ND & 229 & 0 & ND & ND \\
\hline Serratia quinivorans & ND & ND & 227 & 0 & ND & ND \\
\hline Serratia fonticola & 8 & 0 & 114 & 33 & 1047 & 134 \\
\hline Serratia entomophila & 88 & 0 & ND & ND & ND & ND \\
\hline Serratia odorifera & 65 & 0 & 83 & 54 & ND & ND \\
\hline Serratia sp. 506_PEND & ND & ND & 102 & 0 & ND & ND \\
\hline Serratia sp. SCBI & 52 & 2 & 15 & 0 & ND & ND \\
\hline Serratia grimesii & 1 & 0 & 72 & 0 & ND & ND \\
\hline SUM & 25,175 & 32 & 33,993 & 498 & 18,966 & 1830 \\
\hline
\end{tabular}

1 Those values in bold plus labeled with red color represent the top species in the rhizospheric soil of the control cultivar MD12 (MGMRh). ${ }^{2}$ Those species not listed in this table if their mapped read counts in two samples were less than 10 reads. ${ }^{3}$ ND means "not detected".

On the basis of the RefSeq DB, R. aquatilis was identified as the top species with the most mapped read count in the MGMRh sample revealed by One Codex (Table 2, and Table S16 in detail). However, not only Rahnella sp. WP5 and Rahnella sp. Y9602 but also other species of Rahnella were undetected. Meanwhile, S. liquefaciens was undetected (Table 2, and Table S16 in detail), although some other species of Serratia were detected. We suppose that this result might be due to the limited 2948 bacterial genomes in RefSeq DB.

\section{Discussion}

4.1. The Impact of the GT Soybean Line N698 on the Rhizobacteria Has Been Confirmed by 16S rDNA V5-V7 Amplicon Deep Sequencing

In the present study, the box plot analysis of all alpha diversity indices (Figure 1) indicated that the species richness and evenness of the bacterial communities were higher in the rhizospheric soil of N698 than that of MD12, but the difference was not statistically significant (Wilcoxon test). Furthermore, PCoA based on WUF, UUF and Bray-Curtis distance metrics indicated that N698 influenced the beta diversity of the rhizospheric soil bacterial community compared with MD12 (Figure 2). Additionally, some bacterial phyla related to N698 are Proteobacteria, Gemmatimonadetes and Planctomycetes (Table S4). Certain bacterial genera related to N698 are Rahnella, Cellvibrio, Janthinobacterium, Rhodoplanes, Stenotrophomonas, Arthrobacter, Sphingomonas and Nitrospira. (Figure 3A, Table S6). These above results support our previous study on the impact of N698 on the rhizobacteria via $16 \mathrm{~S}$ rDNA V4 hypervariable region amplicon deep sequencing [18].

\subsection{Shortcomings and Solutions of $16 S$ rDNA Amplicon Deep Sequencing}

$16 \mathrm{~S}$ rDNA amplicon deep sequencing via NGS technologies has vastly facilitated the research of complicated microbial communities. However, it still has major technical shortcomings [65], especially 
454 pyrosequencing with low sequencing depth, including biases caused during PCR amplification [66-68], chimeric sequences [68], sequencing errors [68,69] and primer mismatches [70].

Recently, amplicon sequencing errors have decreased to $0.02 \%$ by using effective sequence analysis pipelines $[55,68]$, and low-error $16 \mathrm{~S}$ rDNA amplicon deep sequencing approaches have been developed [71]. Moreover, UCHIME has been developed to significantly reduce chimeric DNA sequences (chimeras) in amplicon deep sequencing data [72]. To reduce biases caused by PCR amplification, Schmidt et al. recommended pooling multiple PCR repeats amplified from the same biological replicate DNA [33]. Furthermore, 16S rDNA fragments generated from Illumina-based SMS is a powerful alternative to $16 \mathrm{~S}$ rDNA amplicons [70]. To evaluate the bias of Illumina-based sequencing of bacterial 16S rDNA amplicon, Kennedy et al. performed PE Illumina sequencing with sufficient depth and found that template concentration exerts a more significant effect on sample profile variability than sample preparation and pooling of multiple PCR amplicons [73].

In addition, the detection limits of $16 \mathrm{~S}$ rDNA amplicon deep sequencing are low reproducibility and quantitation, especially for beta diversity $[74,75]$. Therefore, increasing sampling efforts, including sequencing efforts, and the number of sample replicate are the most effective ways to improve technical reproducibility and quantitation $[65,75]$. Thus, in the present study, an average count per sample with more than 68,996 paired clean reads was near the sequencing number for the desired 90\% OTU overlap.

Some inconsistent results of relative abundances at different classifications between 16S rDNA V4 amplicons in our previous study [18] and V5-V7 amplicon deep sequencing in the present study may be attributed not only to the lack of pooling multiple PCR repeats but also to the different hypervariable regions of $16 \mathrm{~S}$ rDNA. Furthermore, due to short length of $16 \mathrm{~S}$ rDNA amplicon, it is very difficult to distinguish OTU at the genus level, especially at the species level. Deep sequencing full-length 16S rDNA by third-generation sequencing technologies, PacBio single-molecule real-time (SMRT) sequencing system, is a much effective alternative to overcome $16 \mathrm{~S}$ rDNA amplicon PCR biases and primer mismatches [76].

\subsection{Shotgun Metagenome Sequencing via NGS Technology and Bioinformatics Tool}

To identify the richest OTU in the present study, we performed SMS via Illumina Hiseq2500 combined bioinformatics tools instead of deep sequencing full-length 16S rDNA because SMS is not affected by PCR biases or chimeras. In addition, SMS is a powerful method not only for analyzing the entire phylogenetic, taxonomic, genetic and functional diversity of microbial communities but also for discovering new genes, regulators and pathways [65].

However, the large datasets generated by NGS platforms, e.g., 454 GS FLX, Illumina HiSeq/MiSeq, and Ion Torrent PGM, require specialized computing hardware and software and need massive computational resources but still produce relatively short contigs in many studies (reviewed in [65]) and the present study by IDBA-UD [58]. In addition to IDBA-UD, numerous bioinformatics approaches, e.g., CLARK [77], GOTTCHA [78], Kraken [79], and One Codex platform [59] have been developed to explore the taxonomic assignment and functional diversity of complicated microbial metagenomes.

Basing from the evaluation of the accuracy and speed of 14 metagenome bioinformatics tools by Lindgreen et al. [60], we also selected One Codex platform to analyze our metagenomic data and found that One Codex is much more effective and speedy than SOAPaligner (Table S9). Furthermore, taxonomic compositions at the genus level based on One Codex DB by One Codex were consistent with those results based on RefSeq DB by One Codex (Table S17).

Besides deep sequencing full-length 16S rDNA [76], PacBio SMRT has significantly improved the metagenome assembly when it is combined with HiSeq 2000 data [80]. A recent study has noted that MinION, a nanopore-based long-read sequencing platform, followed by Kraken or One Codex bioinformatics analysis, displays the potential to provide accurate and rapid metagenomic analysis [81]. This technology may be the most important advancement in environmental metagenome sequencing and functional discovery, because accurate, long (multi-kb) synthetic reads have detected rare microorganisms and resolved complex populations [82]. 


\subsection{Potential Reason and Consequences of Depletion of Rahnella Genus}

The previous studies have reported that several ways by which transgenic plants affected the structure and function of soil microbes, including horizontal gene transfer from transgenic plant to microbes, [83-86] transgene expression products released from roots or the residues of transgenic plants $[6,87,88]$, and unintentional shifts of root exudate composition in rhizosphere soil of transgenic plants [89-91]. However, we feel it is very hard to analyze the main reason of depletion of Rahnella, because the male parent of the GT soybean line NZL02-92, which was the male parent of N698, was the derivative strain of the GT soybean line AG4501 that was a patented product bred by Asgrow Company, and to date, only a related reference is available from all the databases at Web of Science as follows: “Buettner MJ 1998. New soybean cultivar (9312069421822B) is useful in plant breeding programs to produce superior hybrids. Patent No. US5998704-A.9312069421822B has a higher yield, 73.06 bushels/acre compared to 68.53 bushels/acre for Asgrow AG4501."

In turn, these affected soil microbes, especially rhizosphere microbes, could eventually have impacts on the growth and nutritional status of the transgenic plants. In this study, we found some dominant genera including Rahnella shown to be affected in the rhizosphere soil of the transgenic soybean plants. The potential consequences of depletion of Rahnella could affect the growth, pathogen suppression, and health of the transgenic soybean plant. Since some species or strains of the genus Rahnella have already identified as plant growth-promoting rhizobacteria, for example, some isolates of $R$. aquatilis that reportedly act as a nitrogen fixer [92,93]. Some strains or species of Rahnella genus have the phosphate-solubilizing traits, including a R. aquatilis strain ISL19 [94], and a Rahnella sp. strain isolated from Hippophae rhamnoides rhizosphere [95]. Furthermore, one strain of R. aquatilis was identified as an efficient phytate-degrading rhizobacteria [96]. Moreover, some strain or species of R. aquatilis can inhibit plant pathogen, including a R. aquatilis strain HX2 [97] and a R. aquatilis isolate 36 [98]. Additionally, the endophytic bacterium Rahnella sp. JN6 showed very high Cd, $\mathrm{Pb}$ and $\mathrm{Zn}$ tolerance and plant growth-promoting traits [99]. Thus, these microbial activities just mentioned above like nitrogen-fixing, phosphate-solubilizing, phytate-degrading, inhibition of plant pathogen, and bioremediation will undoubtedly be beneficial for the plant growth.

\section{Conclusions}

Consistent with our previous findings via 16S rDNA V4 region amplicon sequencing, this study showed that the GT soybean line N698 influences the rhizosphere bacterial communities at the flowering stage compared with the cultivar MD12 as control under field conditions via 16S rDNA V5-V7 region amplicon sequencing and shotgun metagenomic approaches. In specific, the GT soybean line N698 negatively affects Rahnella, Janthinobacterium, Stenotrophomonas, Sphingomonas and Luteibacter while positively affects Arthrobacter, Bradyrhizobium, Ramlibacter and Nitrospira.

Supplementary Materials: The following are available online at http:/ /www.mdpi.com/2073-4425/9/4/214/s1, Figure S1: Rarefaction curves of alpha diversity indices of MRh and NRh samples (16S rDNA V5-V7), File S1: OTU_final sequence in MRh and NRh samples (16S rDNA V5-V7), Table S1: Summary of reads, tags and OTUs of MRh and NRh samples (16S rDNA V5-V7), Table S2: Normalized OTU table for biom format of MRh and NRh samples at the flowering stage (16S rDNA V5-V7), Table S3: Comparison of alpha diversity between MRh and NRh based on normalized OTU table (16S rDNA V5-V7), Table S4: Differentially relative abundances of bacterial phyla between MRh and NRh (16S rDNA V5-V7), Table S5: Absolute abundances of bacterial genera in MRh and NRh samples (16S rDNA V5-V7), Table S6: Differentially relative abundances of characterized genera between MRh and NRh (16S rDNA V5-V7), Table S7: Absolute abundances of bacterial species in MRh and NRh samples (16S rDNA V5-V7), Table S8: Top 30 dominant genera in MRh and NRh samples revealed by 16S rDNA amplicon deep sequencing, Table S9: Quality control report and statistical summary of assembled metagenome sequencing data (IDBA + One codex), Table S10: Comparison of mapped reads between MGMRh and MGNRh at all taxonomic levels by SOAPaligner, Table S11: Computation and comparison of mapped reads between MGMRh and MGNRh at all taxonomic levels by One Codex based on One Codex DB, Table S12: Computation and comparison of mapped reads between MGMRh and MGNRh at the genus level by One Codex based on One Codex DB, Table S13: Computation and comparison of mapped reads between MGMRh and MGNRh at all taxonomic levels by One Codex based on RefSeq DB, Table S14: Computation and comparison of mapped reads between MGMRh and MGNRh at the genus level by One Codex based on RefSeq DB, Table S15: Computation 
and comparison of mapped reads between MGMRh and MGNRh at the species level by One Codex based on One Codex DB, Table S16: Computation and comparison of mapped reads between MGMRh and MGNRh at the species level by One Codex based on RefSeq DB, Table S17: Comparison of mapped read count (\%) of top 30 genera in the MGMRh and MGNRh samples by shotgun metagenomic approaches.

Acknowledgments: We thank Aliya Fazal for checking the English language. We are grateful to Long-Guo Jin and Li-Juan Qiu of the Institute of Crop Sciences, the Chinese Academy of Agriculture Sciences (CAAS), for providing soybean seeds of the GT line N698 and its control cultivar MD12. This work was supported by grants from the National Important Science and Technology Specific Project (2016ZX08011-003, 2013ZX08011-003) to Yong-Hua Yang, the National Natural Science Foundation of China (31372140) to Yong-Hua Yang, and the Program for Changjiang Scholars and Innovative Research Team in University from the Ministry of Education of China (IRT_14R27) to Yong-Hua Yang.

Author Contributions: Y.-H.Y., C.-Y.T. and J.-L.Q. conceived and designed the experiments; G.-H.L., X.-M.H., L.L., Z.-L.W. and M.-H.D. performed the experiments; G.-H.L. and X.-M.H. analyzed the data; F.-F.M. and Y.-J.P. contributed reagents and materials; G.-H.L. and X.-M.H. wrote the manuscript; Y.-H.Y., C.-Y.T. and J.-L.Q. revised the manuscript; all authors read and approved the manuscript.

Conflicts of Interest: The authors declare no conflict of interest. The founding sponsors had no role in the design of the study; in the collection, analyses, or interpretation of data; in the writing of the manuscript, and in the decision to publish the results.

\section{References}

1. James, C. Global status of commercialized biotech/GM Crops: 2015. China Biotechnol. 2016, 36, 1-11. [CrossRef]

2. Benbrook, C.M. Trends in glyphosate herbicide use in the United States and globally. Environ. Sci. Eur. 2016, 28, 1-15. [CrossRef] [PubMed]

3. ISAAA. Global status of commercialized biotech/GM Crops: 2016. China Biotechnol. 2017, 37, 1-8. [CrossRef]

4. Dunfield, K.E.; Germida, J.J. Impact of genetically modified crops on soil- and plant-associated microbial communities. J. Environ. Qual. 2004, 33, 806-815. [CrossRef] [PubMed]

5. Liu, B.; Zeng, Q.; Yan, F.M.; Xu, H.G.; Xu, C.R. Effects of transgenic plants on soil microorganisms. Plant Soil 2005, 271, 1-13. [CrossRef]

6. Turrini, A.; Sbrana, C.; Giovannetti, M. Belowground environmental effects of transgenic crops: A soil microbial perspective. Res. Microbiol. 2015, 166, 121-131. [CrossRef] [PubMed]

7. Lugtenberg, B.; Kamilova, F. Plant-growth-promoting rhizobacteria. Annu. Rev. Microbiol. 2009, 63, 541-556. [CrossRef] [PubMed]

8. Berendsen, R.L.; Pieterse, C.M.J.; Bakker, P.A.H.M. The rhizosphere microbiome and plant health. Trends Plant Sci. 2012, 17, 478-486. [CrossRef] [PubMed]

9. Bulgarelli, D.; Schlaeppi, K.; Spaepen, S.; van Themaat, E.V.L.; Schulze-Lefert, P. Structure and functions of the bacterial microbiota of plants. Annu. Rev. Plant Biol. 2013, 64, 807-838. [CrossRef] [PubMed]

10. Souza, R.A.; Babujia, L.C.; Silva, A.P.; Guimaraes, M.d.F.; Arias, C.A.; Hungria, M. Impact of the ahas transgene and of herbicides associated with the soybean crop on soil microbial communities. Transgen. Res. 2013, 22, 877-892. [CrossRef] [PubMed]

11. Liang, J.G.; Sun, S.; Ji, J.; Wu, H.Y.; Meng, F.; Zhang, M.R.; Zheng, X.B.; Wu, C.X.; Zhang, Z.G. Comparison of the rhizosphere bacterial communities of Zigongdongdou soybean and a high-methionine transgenic line of this cultivar. PLoS ONE 2014, 9, e103343. [CrossRef] [PubMed]

12. Sohn, S.I.; Oh, Y.J.; Kim, B.Y.; Cho, H.S. Effects of CaMSRB2-Expressing transgenic rice cultivation on soil microbial communities. J. Microbiol. Biotechnol. 2016, 26, 1303-1310. [CrossRef] [PubMed]

13. Zhou, D.G.; Xu, L.P.; Gao, S.W.; Guo, J.L.; Luo, J.; You, Q.; Que, Y.X. Cry1Ac Transgenic sugarcane does not affect the diversity of microbial communities and has no significant effect on enzyme activities in rhizosphere soil within one crop season. Front. Plant Sci. 2016, 7. [CrossRef] [PubMed]

14. Guan, Z.J.; Lu, S.B.; Huo, Y.L.; Guan, Z.P.; Liu, B.A.; Wei, W. Do genetically modified plants affect adversely on soil microbial communities? Agric. Ecosyst. Environ. 2016, 235, 289-305. [CrossRef]

15. Lee, Y.E.; Yang, S.H.; Bae, T.W.; Kang, H.G.; Lim, P.O.; Lee, H.Y. Effects of field-grown genetically modified zoysia grass on bacterial community structure. J. Microbiol. Biotechnol. 2011, 21, 333-340. [CrossRef] [PubMed] 
16. Hungria, M.; Mendes, I.C.; Nakatani, A.S.; dos Reis-Junior, F.B.; Morais, J.Z.; Neves de Oliveira, M.C.; Fernandes, M.F. Effects of the glyphosate-resistance gene and herbicides on soybean: Field trials monitoring biological nitrogen fixation and yield. Field Crop Res. 2014, 158, 43-54. [CrossRef]

17. Babujia, L.C.; Silva, A.P.; Nakatani, A.S.; Cantao, M.E.; Ribeiro Vasconcelos, A.T.; Visentainer, J.V.; Hungria, M. Impact of long-term cropping of glyphosate-resistant transgenic soybean Glycine max (L.) Merr. on soil microbiome. Transgenic Res. 2016, 25, 425-440. [CrossRef] [PubMed]

18. Lu, G.-H.; Zhu, Y.-L.; Kong, L.-R.; Cheng, J.; Tang, C.-Y.; Hua, X.-M.; Meng, F.-F.; Pang, Y.-J.; Yang, R.-W.; Qi, J.-L.; et al. Impact of a glyphosate-tolerant soybean line on the rhizobacteria, revealed by illumina MiSeq. J. Microbiol. Biotechnol. 2017, 27, 561-572. [CrossRef] [PubMed]

19. Gans, J.; Wolinsky, M.; Dunbar, J. Computational improvements reveal great bacterial diversity and high metal toxicity in soil. Science 2005, 309, 1387-1390. [CrossRef] [PubMed]

20. Tringe, S.G.; von Mering, C.; Kobayashi, A.; Salamov, A.A.; Chen, K.; Chang, H.W.; Podar, M.; Short, J.M.; Mathur, E.J.; Detter, J.C.; et al. Comparative metagenomics of microbial communities. Science 2005, 308, 554-557. [CrossRef] [PubMed]

21. Inceoglu, O.; Abu Al-Soud, W.; Salles, J.F.; Semenov, A.V.; van Elsas, J.D. Comparative analysis of bacterial communities in a potato field as determined by pyrosequencing. PLoS ONE 2011, 6, e23321. [CrossRef] [PubMed]

22. Bulgarelli, D.; Rott, M.; Schlaeppi, K.; van Themaat, E.V.L.; Ahmadinejad, N.; Assenza, F.; Rauf, P.; Huettel, B.; Reinhardt, R.; Schmelzer, E.; et al. Revealing structure and assembly cues for Arabidopsis root-inhabiting bacterial microbiota. Nature 2012, 488, 91-95. [CrossRef] [PubMed]

23. Lundberg, D.S.; Lebeis, S.L.; Paredes, S.H.; Yourstone, S.; Gehring, J.; Malfatti, S.; Tremblay, J.; Engelbrektson, A.; Kunin, V.; del Rio, T.G.; et al. Defining the core Arabidopsis thaliana root microbiome. Nature 2012, 488, 86-90. [CrossRef] [PubMed]

24. Fierer, N.; Lauber, C.L.; Ramirez, K.S.; Zaneveld, J.; Bradford, M.A.; Knight, R. Comparative metagenomic, phylogenetic and physiological analyses of soil microbial communities across nitrogen gradients. ISME J. 2012, 6, 1007-1017. [CrossRef] [PubMed]

25. Sogin, M.L.; Morrison, H.G.; Huber, J.A.; Mark Welch, D.; Huse, S.M.; Neal, P.R.; Arrieta, J.M.; Herndl, G.J. Microbial diversity in the deep sea and the underexplored "rare biosphere". Proc. Natl. Acad. Sci. USA 2006, 103, 12115-12120. [CrossRef] [PubMed]

26. Barriuso, J.; Valverde, J.R.; Mellado, R.P. Effect of Cry1Ab Protein on rhizobacterial communities of Bt-maize over a four-year cultivation period. PLoS ONE 2012, 7, e35481. [CrossRef] [PubMed]

27. Verbruggen, E.; Kuramae, E.E.; Hillekens, R.; de Hollander, M.; Kiers, E.T.; Roling, W.F.M.; Kowalchuk, G.A.; van der Heijden, M.G.A. Testing potential effects of maize expressing the Bacillus thuringiensis Cry1 $\mathrm{Ab}$ Endotoxin (BT Maize) on mycorrhizal fungal communities via DNA- and RNA-Based pyrosequencing and molecular fingerprinting. Appl. Environ. Microbiol. 2012, 78, 7384-7392. [CrossRef] [PubMed]

28. Kuramae, E.E.; Verbruggen, E.; Hillekens, R.; de Hollander, M.; Roling, W.F.M.; van der Heijden, M.G.A.; Kowalchuk, G.A. Tracking Fungal Community Responses to Maize Plants by DNA- and RNA-Based Pyrosequencing. PLoS ONE 2013, 8, e69973. [CrossRef] [PubMed]

29. Caporaso, J.G.; Lauber, C.L.; Walters, W.A.; Berg-Lyons, D.; Lozupone, C.A.; Turnbaugh, P.J.; Fierer, N.; Knight, R. Global patterns of $16 \mathrm{~S}$ rRNA diversity at a depth of millions of sequences per sample. Proc. Natl. Acad. Sci. USA 2011, 108, 4516-4522. [CrossRef] [PubMed]

30. Caporaso, J.G.; Lauber, C.L.; Walters, W.A.; Berg-Lyons, D.; Huntley, J.; Fierer, N.; Owens, S.M.; Betley, J.; Fraser, L.; Bauer, M.; et al. Ultra-high-throughput microbial community analysis on the Illumina HiSeq and MiSeq platforms. ISME J. 2012, 6, 1621-1624. [CrossRef] [PubMed]

31. Kozich, J.J.; Westcott, S.L.; Baxter, N.T.; Highlander, S.K.; Schloss, P.D. Development of a dual-index sequencing strategy and curation pipeline for analyzing amplicon sequence data on the MiSeq Illumina Sequencing Platform. Appl. Environ. Microbiol. 2013, 79, 5112-5120. [CrossRef] [PubMed]

32. Fierer, N.; Leff, J.W.; Adams, B.J.; Nielsen, U.N.; Bates, S.T.; Lauber, C.L.; Owens, S.; Gilbert, J.A.; Wall, D.H.; Caporaso, J.G. Cross-biome metagenomic analyses of soil microbial communities and their functional attributes. Proc. Natl. Acad. Sci. USA 2012, 109, 21390-21395. [CrossRef] [PubMed]

33. Schmidt, P.A.; Balint, M.; Greshake, B.; Bandow, C.; Rombke, J.; Schmitt, I. Illumina metabarcoding of a soil fungal community. Soil Biol. Biochem. 2013, 65, 128-132. [CrossRef] 
34. Xian, L.J.; Li, Y.; Jiang, Z.; Ma, J.D.; Jin, L.; Chen, L.; Zhou, C.W.; Zhang, J.; Liu, Y.K.; Zhu, L.; et al. Alterations in cecal microbiota of Jinhua piglets fostered by a Yorkshire sow. Chin. Sci. Bull. 2014, 59, 4304-4311. [CrossRef]

35. Yang, C.Y.; Li, Y.; Zhou, B.; Zhou, Y.Y.; Zheng, W.; Tian, Y.; Van Nostrand, J.D.; Wu, L.Y.; He, Z.L.; Zhou, J.Z.; et al. Illumina sequencing-based analysis of free-living bacterial community dynamics during an Akashiwo sanguine bloom in Xiamen sea, China. Sci. Rep. 2015, 5, 8476. [CrossRef] [PubMed]

36. Edwards, J.; Johnson, C.; Santos-Medellin, C.; Lurie, E.; Podishetty, N.K.; Bhatnagar, S.; Eisen, J.A.; Sundaresan, V. Structure, variation, and assembly of the root-associated microbiomes of rice. Proc. Natl. Acad. Sci. USA 2015, 112, E911-E920. [CrossRef] [PubMed]

37. Mashiane, R.A.; Ezeokoli, O.T.; Adeleke, R.A.; Bezuidenhout, C.C. Metagenomic analyses of bacterial endophytes associated with the phyllosphere of a BT maize cultivar and its isogenic parental line from South Africa. World J. Microbiol. Biotechnol. 2017, 33, 80. [CrossRef] [PubMed]

38. Zhu, W.X.; Chu, Y.G.; Ding, C.J.; Huang, Q.J.; Zhang, B.Y.; Zhang, W.X.; Su, X.H. Assessing bacterial communities in the rhizosphere of 8-year-old genetically modified poplar (Populus spp.). J. For. Res. 2016, 27, 939-947. [CrossRef]

39. Debruyn, J.M.; Bevard, D.A.; Essington, M.E.; McKnight, J.Y.; Schaeffer, S.M.; Baxter, H.L.; Mazarei, M.; Mann, D.G.J.; Dixon, R.A.; Chen, F.; et al. Field-grown transgenic switchgrass (Panicum virgatum L.) with altered lignin does not affect soil chemistry, microbiology, and carbon storage potential. Glob. Chang. Biol. Bioenergy 2017, 9, 1100-1109. [CrossRef]

40. Hess, M.; Sczyrba, A.; Egan, R.; Kim, T.W.; Chokhawala, H.; Schroth, G.; Luo, S.J.; Clark, D.S.; Chen, F.; Zhang, T; et al. Metagenomic discovery of biomass-degrading genes and genomes from cow rumen. Science 2011, 331, 463-467. [CrossRef] [PubMed]

41. Yu, K.; Zhang, T. Metagenomic and Metatranscriptomic analysis of microbial community structure and gene expression of activated sludge. PLoS ONE 2012, 7, e38183. [CrossRef] [PubMed]

42. Le Chatelier, E.; Nielsen, T.; Qin, J.J.; Prifti, E.; Hildebrand, F.; Falony, G.; Almeida, M.; Arumugam, M.; Batto, J.M.; Kennedy, S.; et al. Richness of human gut microbiome correlates with metabolic markers. Nature 2013, 500, 541-546. [CrossRef] [PubMed]

43. Bremges, A.; Maus, I.; Belmann, P.; Eikmeyer, F.; Winkler, A.; Albersmeier, A.; Puhler, A.; Schluter, A.; Sczyrba, A. Deeply sequenced metagenome and metatranscriptome of a biogas-producing microbial community from an agricultural production-scale biogas plant. Gigascience 2015, 4, 33. [CrossRef] [PubMed]

44. Mangrola, A.; Dudhagara, P.; Koringa, P.; Joshi, C.G.; Parmar, M.; Patel, R. Deciphering the microbiota of Tuwa hot spring, India using shotgun metagenomic sequencing approach. Genom. Data 2015, 4, $153-155$. [CrossRef] [PubMed]

45. Mangrola, A.V.; Dudhagara, P.; Koringa, P.; Joshi, C.G.; Patel, R.K. Shotgun metagenomic sequencing based microbial diversity assessment of Lasundra hot spring, India. Genom. Data 2015, 4, 73-75. [CrossRef] [PubMed]

46. Tsurumaru, H.; Okubo, T.; Okazaki, K.; Hashimoto, M.; Kakizaki, K.; Hanzawa, E.; Takahashi, H.; Asanome, N.; Tanaka, F.; Sekiyama, Y.; et al. Metagenomic analysis of the bacterial community associated with the taproot of sugar beet. Microbes Environ. 2015, 30, 63-69. [CrossRef] [PubMed]

47. Hultman, J.; Waldrop, M.P.; Mackelprang, R.; David, M.M.; McFarland, J.; Blazewicz, S.J.; Harden, J.; Turetsky, M.R.; McGuire, A.D.; Shah, M.B.; et al. Multi-omics of permafrost, active layer and thermokarst bog soil microbiomes. Nature 2015, 521, 208-212. [CrossRef] [PubMed]

48. Nesme, J.; Cecillon, S.; Delmont, T.O.; Monier, J.M.; Vogel, T.M.; Simonet, P. Large-scale metagenomic-based study of antibiotic resistance in the environment. Curr. Biol. 2014, 24, 1096-1100. [CrossRef] [PubMed]

49. Zhang, X.; Zhang, D.Y.; Jia, H.J.; Feng, Q.; Wang, D.H.; Liang, D.; Wu, X.N.; Li, J.H.; Tang, L.Q.; Li, Y.; et al. The oral and gut microbiomes are perturbed in rheumatoid arthritis and partly normalized after treatment. Nat. Med. 2015, 21, 895-905. [CrossRef] [PubMed]

50. Jalali, S.; Kohli, S.; Latka, C.; Bhatia, S.; Vellarikal, S.K.; Sivasubbu, S.; Scaria, V.; Ramachandran, S. Screening currency notes for microbial pathogens and antibiotic resistance genes using a shotgun metagenomic approach. PLoS ONE 2015, 10, e0128711. [CrossRef] [PubMed]

51. Chauhan, A.; Smartt, A.; Wang, J.; Utturkar, S.; Frank, A.; Bi, M.; Liu, J.; Williams, D.; Xu, T.; Eldridge, M.; et al. Integrated metagenomics and metatranscriptomics analyses of root-associated soil from transgenic switchgrass. Genome Announc. 2014, 2. [CrossRef] [PubMed] 
52. Hily, J.-M.; Demaneche, S.; Poulicard, N.; Tannieres, M.; Djennane, S.; Beuve, M.; Vigne, E.; Demangeat, G.; Komar, V.; Gertz, C.; et al. Metagenomic-based impact study of transgenic grapevine rootstock on its associated virome and soil bacteriome. Plant Biotechnol. J. 2018, 16. [CrossRef] [PubMed]

53. Fadrosh, D.W.; Ma, B.; Gajer, P.; Sengamalay, N.; Ott, S.; Brotman, R.M.; Ravel, J. An improved dual-indexing approach for multiplexed $16 \mathrm{~S}$ rRNA gene sequencing on the Illumina MiSeq platform. Microbiome 2014, 2, 6. [CrossRef] [PubMed]

54. Schlaeppi, K.; Dombrowski, N.; Oter, R.G.; van Themaat, E.V.L.; Schulze-Lefert, P. Quantitative divergence of the bacterial root microbiota in Arabidopsis thaliana relatives. Proc. Natl. Acad. Sci. USA 2014, 111, 585-592. [CrossRef] [PubMed]

55. Edgar, R.C. UPARSE: Highly accurate OTU sequences from microbial amplicon reads. Nat. Methods 2013, 10, 996-998. [CrossRef] [PubMed]

56. Caporaso, J.G.; Kuczynski, J.; Stombaugh, J.; Bittinger, K.; Bushman, F.D.; Costello, E.K.; Fierer, N.; Pena, A.G.; Goodrich, J.K.; Gordon, J.I.; et al. QIIME allows analysis of high-throughput community sequencing data. Nat Methods 2010, 7, 335-336. [CrossRef] [PubMed]

57. Qin, J.J.; Li, Y.R.; Cai, Z.M.; Li, S.H.; Zhu, J.F.; Zhang, F.; Liang, S.S.; Zhang, W.W.; Guan, Y.L.; Shen, D.Q.; et al. A metagenome-wide association study of gut microbiota in type 2 diabetes. Nature 2012, 490, 55-60. [CrossRef] [PubMed]

58. Peng, Y.; Leung, H.C.; Yiu, S.M.; Chin, F.Y. IDBA-UD: A de novo assembler for single-cell and metagenomic sequencing data with highly uneven depth. Bioinformatics 2012, 28, 1420-1428. [CrossRef] [PubMed]

59. Minot, S.S.; Krumm, N.; Greenfield, N.B. One Codex: A Sensitive and accurate data platform for genomic microbial identification. bioRxiv 2015, 23. [CrossRef]

60. Lindgreen, S.; Adair, K.L.; Gardner, P.P. An evaluation of the accuracy and speed of metagenome analysis tools. Sci. Rep. 2016, 6, 19233. [CrossRef] [PubMed]

61. Li, R.Q.; Yu, C.; Li, Y.R.; Lam, T.W.; Yiu, S.M.; Kristiansen, K.; Wang, J. SOAP2: An improved ultrafast tool for short read alignment. Bioinformatics 2009, 25, 1966-1967. [CrossRef] [PubMed]

62. White, J.R.; Nagarajan, N.; Pop, M. Statistical Methods for Detecting differentially abundant features in clinical metagenomic samples. PLoS Comput. Biol. 2009, 5, e1000352. [CrossRef] [PubMed]

63. Lu, G.-H.; Hua, X.-M.; Cheng, J.; Zhu, Y.-L.; Wang, G.-H.; Pang, Y.-J.; Yang, R.-W.; Zhang, L.; Shou, H.; Wang, X.-M.; et al. Impact of glyphosate on the rhizosphere microbial communities of an EPSPS-Transgenic Soybean Line ZUTS31 by metagenome sequencing. Curr. Genom. 2018, 19, 34-49. [CrossRef] [PubMed]

64. Benjamini, Y.; Hochberg, Y. Controlling the false discovery rate - a practical and powerful approach to multiple testing. J. R. Stat. Soc. Series B Stat. Methodol. 1995, 57, 289-300.

65. Zhou, J.Z.; He, Z.L.; Yang, Y.F.; Deng, Y.; Tringe, S.G.; Alvarez-Cohen, L. High-Throughput metagenomic technologies for complex microbial community analysis: Open and closed formats. mBio 2015, 6, e02288-14. [CrossRef] [PubMed]

66. Lemos, L.N.; Fulthorpe, R.R.; Roesch, L.F.W. Low sequencing efforts bias analyses of shared taxa in microbial communities. Folia Microbiol. 2012, 57, 409-413. [CrossRef] [PubMed]

67. Pinto, A.J.; Raskin, L. PCR Biases Distort bacterial and archaeal community structure in pyrosequencing datasets. PLoS ONE 2012, 7, e43093. [CrossRef] [PubMed]

68. Schloss, P.D.; Gevers, D.; Westcott, S.L. Reducing the effects of PCR amplification and sequencing artifacts on 16S rRNA-based studies. PLoS ONE 2011, 6, e27310. [CrossRef] [PubMed]

69. Kunin, V.; Engelbrektson, A.; Ochman, H.; Hugenholtz, P. Wrinkles in the rare biosphere: Pyrosequencing errors can lead to artificial inflation of diversity estimates. Environ. Microbiol. 2010, 12, 118-123. [CrossRef] [PubMed]

70. Logares, R.; Sunagawa, S.; Salazar, G.; Cornejo-Castillo, F.M.; Ferrera, I.; Sarmento, H.; Hingamp, P.; Ogata, H.; de Vargas, C.; Lima-Mendez, G.; et al. Metagenomic 16S rDNA Illumina tags are a powerful alternative to amplicon sequencing to explore diversity and structure of microbial communities. Environ. Microbiol. 2014, 16, 2659-2671. [CrossRef] [PubMed]

71. Lundberg, D.S.; Yourstone, S.; Mieczkowski, P.; Jones, C.D.; Dangl, J.L. Practical innovations for high-throughput amplicon sequencing. Nat. Methods 2013, 10, 999-1002. [CrossRef] [PubMed]

72. Edgar, R.C.; Haas, B.J.; Clemente, J.C.; Quince, C.; Knight, R. UCHIME improves sensitivity and speed of chimera detection. Bioinformatics 2011, 27, 2194-2200. [CrossRef] [PubMed] 
73. Kennedy, K.; Hall, M.W.; Lynch, M.D.J.; Moreno-Hagelsieb, G.; Neufeld, J.D. Evaluating bias of Illumina-based bacterial 16S rRNA gene profiles. Appl. Environ. Microbiol. 2014, 80, 5717-5722. [CrossRef] [PubMed]

74. Zhou, J.; Wu, L.; Deng, Y.; Zhi, X.; Jiang, Y.H.; Tu, Q.; Xie, J.; Van Nostrand, J.D.; He, Z.; Yang, Y. Reproducibility and quantitation of amplicon sequencing-based detection. ISME J. 2011, 5, 1303-1313. [CrossRef] [PubMed]

75. Zhou, J.; Jiang, Y.H.; Deng, Y.; Shi, Z.; Zhou, B.Y.; Xue, K.; Wu, L.; He, Z.; Yang, Y. Random sampling process leads to overestimation of $\beta$-diversity of microbial communities. mBio 2013, 4, e00324-13. [CrossRef] [PubMed]

76. Pootakham, W.; Mhuantong, W.; Yoocha, T.; Putchim, L.; Sonthirod, C.; Naktang, C.; Thongtham, N.; Tangphatsornruang, S. High resolution profiling of coral-associated bacterial communities using full-length 16S rRNA sequence data from PacBio SMRT sequencing system. Sci. Rep. 2017, 7, 2774. [CrossRef] [PubMed]

77. Ounit, R.; Wanamaker, S.; Close, T.J.; Lonardi, S. CLARK: Fast and accurate classification of metagenomic and genomic sequences using discriminative k-mers. BMC Genom. 2015, 16, 236. [CrossRef] [PubMed]

78. Freitas, T.A.K.; Li, P.E.; Scholz, M.B.; Chain, P.S.G. Accurate read-based metagenome characterization using a hierarchical suite of unique signatures. Nucleic Acids Res. 2015, 43, e69. [CrossRef] [PubMed]

79. Wood, D.E.; Salzberg, S.L. Kraken: Ultrafast metagenomic sequence classification using exact alignments. Genome Biol. 2014, 15, R46. [CrossRef] [PubMed]

80. Frank, J.A.; Pan, Y.; Tooming-Klunderud, A.; Eijsink, V.G.; McHardy, A.C.; Nederbragt, A.J.; Pope, P.B. Improved metagenome assemblies and taxonomic binning using long-read circular consensus sequence data. Sci. Rep. 2016, 6, 25373. [CrossRef] [PubMed]

81. Brown, B.L.; Watson, M.; Minot, S.S.; Rivera, M.C.; Franklin, R.B. MinION (TM) nanopore sequencing of environmental metagenomes: A synthetic approach. Gigascience 2017, 6. [CrossRef] [PubMed]

82. Sharon, I.; Kertesz, M.; Hug, L.A.; Pushkarev, D.; Blauwkamp, T.A.; Castelle, C.J.; Amirebrahimi, M.; Thomas, B.C.; Burstein, D.; Tringe, S.G.; et al. Accurate, multi-kb reads resolve complex populations and detect rare microorganisms. Genome Res. 2015, 25, 534-543. [CrossRef] [PubMed]

83. de Vries, J.; Herzfeld, T.; Wackernagel, W. Transfer of plastid DNA from tobacco to the soil bacterium Acinetobacter sp. by natural transformation. Mol. Microbiol. 2004, 53, 323-334. [CrossRef] [PubMed]

84. Simpson, D.J.; Fry, J.C.; Rogers, H.J.; Day, M.J. Transformation of Acinetobacter baylyi in non-sterile soil using recombinant plant nuclear DNA. Environ. Biosaf. Res. 2007, 6, 101-112. [CrossRef] [PubMed]

85. Rizzi, A.; Pontiroli, A.; Brusetti, L.; Borin, S.; Sorlini, C.; Abruzzese, A.; Sacchi, G.A.; Vogel, T.M.; Simonet, P.; Bazzicalupo, M.; et al. Strategy for in situ detection of natural transformation-based horizontal gene transfer events. Appl. Environ. Microbiol. 2008, 74, 1250-1254. [CrossRef] [PubMed]

86. Pontiroli, A.; Rizzi, A.; Simonet, P.; Daffonchio, D.; Vogel, T.M.; Monier, J.M. Visual evidence of horizontal gene transfer between plants and bacteria in the phytosphere of transplastomic tobacco. Appl. Environ. Microbiol. 2009, 75, 3314-3322. [CrossRef] [PubMed]

87. Ahrenholtz, I.; Harms, K.; de Vries, J.; Wackernagel, W. Increased killing of Bacillus subtilis on the hair roots of transgenic t4 lysozyme-producing potatoes. Appl. Environ. Microbiol. 2000, 66, 1862-1865. [CrossRef] [PubMed]

88. Saxena, D.; Flores, S.; Stotzky, G. Bt toxin is released in root exudates from 12 transgenic corn hybrids representing three transformation events. Soil Biol. Biochem. 2002, 34, 133-137. [CrossRef]

89. Tesfaye, M.; Dufault, N.S.; Dornbusch, M.R.; Allan, D.L.; Vance, C.P.; Samac, D.A. Influence of enhanced malate dehydrogenase expression by alfalfa on diversity of rhizobacteria and soil nutrient availability. Soil Biol. Biochem. 2003, 35, 1103-1113. [CrossRef]

90. Poerschmann, J.; Gathmann, A.; Augustin, J.; Langer, U.; Gorecki, T. Molecular composition of leaves and stems of genetically modified BT and near-isogenic non-BT maize-Characterization of lignin patterns. J. Environ. Qual. 2005, 34, 1508-1518. [CrossRef] [PubMed]

91. Flores, S.; Saxena, D.; Stotzky, G. Transgenic BT plants decompose less in soil than non-BT plants. Soil Biol. Biochem. 2005, 37, 1073-1082. [CrossRef]

92. Berge, O.; Heulin, T.; Achouak, W.; Richard, C.; Bally, R.; Balandreau, J. Rahnella aquatilis—A nitrogen-fixing enteric bacterium associated with the rhizosphere of wheat and maize. Can. J. Microbiol. 1991, 37, 195-203. [CrossRef] 
93. Heulin, T.; Berge, O.; Mavingui, P.; Gouzou, L.; Hebbar, K.P.; Balandreau, J. Bacillus polymyxa and Rahnella aquatilis, the dominant N2-fixing bacteria associated with wheat rhizosphere in French soils. Eur. J. Soil Biol. 1994, 30, 35-42.

94. Kim, K.Y.; Jordan, D.; Krishnan, H.B. Rahnella aquatilis, a bacterium isolated from soybean rhizosphere, can solubilize hydroxyapatite. FEMS Microbiol. Lett. 1997, 153, 273-277. [CrossRef]

95. Vyas, P.; Joshi, R.; Sharma, K.C.; Rahi, P.; Gulati, A.; Gulati, A. Cold-adapted and rhizosphere-competent strain of Rahnella sp. with broad-spectrum plant growth-promotion potential. 2010, 20, 1724-1734. [CrossRef]

96. Li, G.E.; Wu, X.Q.; Ye, J.R.; Hou, L.; Zhou, A.D.; Zhao, L. Isolation and identification of phytate-degrading rhizobacteria with activity of improving growth of poplar and masson pine. World J. Microbiol. Biotechnol. 2013, 29, 2181-2193. [CrossRef] [PubMed]

97. Chen, F.; Guo, Y.B.; Wang, J.H.; Li, J.Y.; Wang, H.M. Biological control of grape crown gall by Rahnella aquatilis HX2. Plant Dis. 2007, 91, 957-963. [CrossRef]

98. Palmieri, D.; Vitullo, D.; De Curtis, F.; Lima, G. A microbial consortium in the rhizosphere as a new biocontrol approach against Fusarium decline of chickpea. Plant Soil 2017, 412, 425-439. [CrossRef]

99. He, H.D.; Ye, Z.H.; Yang, D.J.; Yan, J.L.; Xiao, L.; Zhong, T.; Yuan, M.; Cai, X.D.; Fang, Z.Q.; Jing, Y.X. Characterization of endophytic Rahnella sp JN6 from Polygonum pubescens and its potential in promoting growth and Cd, Pb, Zn uptake by Brassica napus. Chemosphere 2013, 90, 1960-1965. [CrossRef] [PubMed]

(C) 2018 by the authors. Licensee MDPI, Basel, Switzerland. This article is an open access article distributed under the terms and conditions of the Creative Commons Attribution (CC BY) license (http://creativecommons.org/licenses/by/4.0/). 